\title{
Helicobacter pylori GroEL Seropositivity Is Associated with an Increased Risk of Opisthorchis viverrini-Associated Hepatobiliary Abnormalities and Cholangiocarcinoma
}

\author{
Isabelle Jala ${ }^{1,2}$, Muhammad Luthfi Almanfaluthi ${ }^{1,2}$, Thewarach Laha ${ }^{3}$, Sakawrat Kanthawong ${ }^{4}$, \\ Sirikachorn Tangkawattana ${ }^{2,5}$, Prasert Saichua ${ }^{1,2}$, Sutas Suttiprapa ${ }^{1,2, *} \mathbb{E}$, Banchob Sripa ${ }^{2,6}$ \\ 'Tropical Medicine Graduate Program (International Program), Academic Affairs, Faculty of Medicine, Khon Kaen University, Khon Kaen, Thailand; \\ ${ }^{2}$ WHO Collaborating Centre for Research and Control of Opisthorchiasis (Southeast Asian Liver Fluke Disease), Tropical Disease Research Centre, \\ Faculty of Medicine, Khon Kaen University, Khon Kaen, Thailand; ${ }^{3}$ Department of Parasitology, Faculty of Medicine, Khon Kaen University, Khon \\ Kaen, Thailand; ' ${ }^{4}$ Department of Microbiology, Faculty of Medicine, Khon Kaen University, Khon Kaen, Thailand; ${ }^{5}$ Department of Pathobiology, \\ Faculty of Veterinary Medicine, Khon Kaen University, Khon Kaen, Thailand; ${ }^{6}$ Department of Pathology, Faculty of Medicine, Khon Kaen University, \\ Khon Kaen, Thailand
}

\begin{abstract}
Despite the synergistic effect of Opisthorchis viverrini and Helicobacter pylori co-infection on pathogenesis of severe hepatobiliary abnormalities (HBA) including advanced periductal fibrosis and replace with cholangiocarcinoma (CCA) have been established, the immune response to $H$. pylori in $O$. viverrini infected population has never been explored. Hence, this study aimed to investigate the antibody responses to 2 immunogenic $H$. pylori proteins in $O$. viverriniinfected patients with HBA and CCA. The risk analysis by multinomial logistic regression revealed that GroEL seropositivity was associated with higher risks of hepatobiliary abnormalities and CCA with adjusted odds ratios $(95 \%$ confidence intervals) of $2.11(95 \% \mathrm{Cl}=1.20-3.71, P=0.008)$ and $2.13(95 \% \mathrm{Cl}=1.21-3.75, P=0.009)$, respectively. These findings indicate that GroEL seropositivity might be a biomarker for early detection of $O$. viverrini associated HBA and CCA.
\end{abstract}

Key words: Opisthorchis viverrini, Helicobacter pylori, risk factor, cholangiocarcinoma, hepatobiliary abnormality

\section{INTRODUCTION}

Infection with the liver fluke Opisthorchis viverrini is associated with the development of hepatobiliary abnormalities (HBA), including the bile duct cancer-cholangiocarcinoma (CCA) [1,2]. The incidence of CCA in the Northeast Thailand is the highest worldwide with 85 cases per 100,000 population [3]. CCA is associated with poor prognosis as most confirmed cases in the Northeast Thailand are stage IV cancers [4]. One major challenge is still early detection of the cancer as it would be associated with a better prognostic [5].

Among other factors, a carcinogenic bacterium, Helicobacter pylori, has been proposed to play an important role in carcinogenesis of CCA [6]. Previous studies demonstrated that O. viverrini is a reservoir host of $H$. pylori [7]. As a result, the preva-

\footnotetext{
- Received 22 April 2021, revised 1 August 2021, accepted 2 August 2021.

*Corresponding author (sutasu@kku.ac.th)

(c) 2021, Korean Society for Parasitology and Tropical Medicine

This is an Open Access article distributed under the terms of the Creative Commons Attribution Non-Commercial License (https://creativecommons.org/licenses/by-nc/4.0) which permits unrestricted non-commercial use, distribution, and reproduction in any medium, provided the original work is properly cited.
}

lence of $H$. pylori infection was higher in $O$. viverrini-infected than uninfected populations [8]. Moreover, severe biliary periductal fibrosis during opisthorchiasis is associated with CagApositive H. pylori infection [8]. Furthermore, co-infection of $O$. viverrini and $H$. pylori enhanced the severity of hepatobiliary abnormalities [9]. These findings support the hypothesis that $H$. pylori contributes to the pathogenesis of chronic opisthorchiasis and opisthorchiasis-associated CCA [10].

Antibody responses to $H$. pylori proteins were demonstrated to be associated with several cancers. For example, previous studies on gastric cancers showed that, among other proteins, seroreactivity to CagA and GroEL proteins of $H$. pylori was associated with cancer risk in a German cohort [11], a Swedish cohort [12], and East Asian cohorts [13]. In addition, seroreactivity to GroEL was associated with a higher risk of colorectal cancer in the US and Spain cohorts $[14,15]$ and with a higher risk of hepatocellular carcinoma in a study on hepatobiliary cancers among Finnish people [16].

Despite the fact that the association between the 2 carcinogenic pathogens $O$. viverrini and $H$. pylori is now established, the immune response to $H$. pylori in O. viverrini-associated dis- 
eases has never been explored. Hence, this study focused on the detection of $\operatorname{IgG}$ antibodies to $2 \mathrm{H}$. pylori immunogenic antigens (CagA and GroEL). A case-control study was designed to determine the significance of antibody response in association with the risk of developing HBA and CCA in an O. viverrini-endemic area.

\section{MATERIALS AND METHODS}

\section{Ethics statement}

This study was approved by the Institutional Human Ethics Committee of Khon Kaen University (HE 611280). Informed consent was obtained from each participant and patients enrolled in this study.

\section{Study design and research subjects}

This study was designed to analyse the $\operatorname{IgG}$ responses to $2 \mathrm{H}$. pylori antigens (virulent factor CagA and chaperonin GroEL) in serum samples collected from a community-based opisthorchiasis control in Northeast Thailand. Overall, 551 samples from patients aged between 30 and 74 years-old, were retrieved from the biobank of the Tropical Disease Research Center (TDRC, Academic Affairs, Faculty of Medicine, Khon Kaen University, Thailand). One hundred and twenty-two $O$. viverrini-negative people without hepatobiliary abnormalities were defined as controls and age- and sex-matched with $140 \mathrm{O}$. viverrini-positive patients without HBA, 144 O. viverrini-positive patients with HBA, and 145 patients with confirmed cholangiocarcinoma. Patients were selected among those who underwent stool examination by a quantitative formalin/ethyl acetate concentration technique for the evaluation of Opisthorchis viverrini infection. Among O. viverrini-positive patients, abdominal ultrasonography by a mobile high-resolution ultrasound machine was used to determine the presence of hepatobiliary abnormalities. These abnormalities included periportal fibrosis of the liver parenchyma, gallbladder wall and size, sludge and suspected cholangiocarcinoma (dilated bile duct and/or liver mass). Fibrosis grading is referring to periportal echoes in 2 to 3 segments or the liver $(\mathrm{HBA}+2)$ or more than 3 $(\mathrm{HBA}+3)$ [17]. Histologically confirmed CCA cases were selected among patients who underwent surgery at Srinagarind Hospital, Faculty of Medicine, Khon Kaen University, Khon Kaen, Thailand.

\section{Gene cloning and protein expression of partial cagA (pcagA) and groEL}

H. pylori cagA and groEL genes were amplified from virulent strains isolated from Thai patients (provided by Professor Ratha-Korn Vilaichone, Thammasat University, Thailand). The details of gene cloning and protein expression of partial CagA (pCagA) and GroEL were described in the Supplementary Data S1 and Supplementary Table S1. Proteins were produced as soluble proteins of 60-65 kDa for GroEL and pCagA (Supplementary Fig. S1).

\section{pCagA and GroEL in-house indirect ELISA}

After validation of positive and negative control sera with the commercial antibody determination kit E-Plate Eiken $H$. pylori antibody (Eiken Chemical Co., Ltd., Tokyo, Japan) and checkerboard titration of reagents, the following conditions were used. The microwell plates (Thermo Fisher Scientific, Waltham, Massachusetts, USA) were coated with $0.05 \mu \mathrm{g}$ proteins/well in sodium phosphate buffer $(90 \mathrm{mM}$ disodium phosphate, $130 \mathrm{mM}$ monosodium phosphate, $\mathrm{pH}$ 6.5) overnight at $4^{\circ} \mathrm{C}$. Wells were washed with $0.05 \%$ Tween 20 in PBS and blocked with $1 \%$ BSA in PBS for $2 \mathrm{hr}$. After washing, serum samples at 1:400 dilution in 1\% BSA in PBS were added to individual wells in duplicate and incubated for $2 \mathrm{hr}$. After washing, a goat anti-human IgG-HRP (Thermo Fisher Scientific) was added at 1:60,000 in PBS for $1 \mathrm{hr}$. The wells were washed, and color reactions were developed with 1-Step Ultra TMB-ELISA Substrate Solution (Thermo Fisher Scientific). After 30 min incubation in the dark, reactions were stopped with $0.2 \mathrm{M}$ sulphuric acid and plates read at $450 \mathrm{~nm}$. Absorbance values (ODs) for all samples were calculated from the mean of 2 measurements and adjusted for interplate variation by dividing the ODs by the positive control OD of each corresponding experiment. The cut-off for positivity was calculated for each protein from the mean of the negative control ODs tested in all plates plus 3 SDs, to exclude positive outliers. Therefore, the negative threshold was 0.175 OD for pCagA ELISA experiments and 0.235 for GroEL experiments. ODs were corrected by subtracting each adjusted sample OD by the negative threshold. In addition, pCagA and GroEL serostatus were determined as follows: negative for corrected ODs equal to or less than 0 ; positive for all values strictly superior to 0 .

\section{Statistical analysis}

All analyses were performed with RStudio software [18]. 
Correlation between anti-H. pylori antigens was analysed by Pearson's correlation (package stats, version 3.5.1, [19]). Results were plotted using raster package version 2.6-7 [20]. Correlations between intensity of O. viverrini infection (EPG levels) and pCagA and GroEL antibody levels were analysed by Kruskal-Wallis test (package stats, version 3.5.1, [19]). Excluding CCA patients for lack of data, the intensity of $O$. viverrini infection was assessed based on egg count (egg per gram of feces, $\mathrm{EPG}$ ): OV negative for $\mathrm{EPG}=0$; light infection for $\mathrm{EPG}<$ 500; moderate for $500>\mathrm{EPG}<1,000$; and heavy for $>1,000$ EPG. Corrected sample ODs from pCagA and GroEL ELISA experiments were categorized in 5 groups: non-responders for $\mathrm{OD}=0$ and low, moderate, high or very higher responders for $\mathrm{OD}>0$ divided by quartiles. Results were plotted with ggplot2 package version 3.2.1 [21]. Risk analysis for O. viverrini infection, hepatobiliary abnormalities and cholangiocarcinoma was performed in association with GroEL and pCagA IgG serostatus by multinomial logistic regression (package nnet, version 7.3-12, [22]). Models were adjusted for age and sex and OV negative group was used as reference group.

\section{RESULTS}

\section{Demographic data}

Details of the tested population are listed in Supplementary Table S2. As samples were adjusted for age and sex among the groups, males constitute 53.3 to $55.2 \%$ of all samples in each group, and the mean age varied between 50.5- and 54.1-yearold. Among the 144 samples from $\mathrm{OV}+\mathrm{HBA}+$ patients, $52.1 \%$ presented HBA in 2 fragments of the liver while the remaining patients showed HBA in 3 fragments of the liver. Among CCA patients, the main represented cancers were well- (31\%), moderately-differentiated adenocarcinomas (22.7\%), and papillary adenocarcinomas $(25.5 \%)$, followed by poorly differentiated adenocarcinomas $(15.9 \%)$, adenosquamous carcinomas (3.5\%) and squamous cell carcinomas (1.4\%). Concerning cancer growth type, a mass-forming type (51\%) was the most common type among patients. The growth type data were missing in $17.2 \%$ of all CCA patients.

\section{Correlation between pCagA with GroEL IgG responses}

Among all 551 samples, 19.2\% were pCagA seronegative and $24.7 \%$ were GroEL seronegative. For pCagA, OD values of seropositive samples ranged from 0.0003 to $1.5 \mathrm{OD}$ with a median of 0.2 . For GroEL, OD ranged from 0.0023 to 1.8 (median OD: 0.3). Comparing pCagA with GroEL seropositive samples, a weak positive correlation was observed between pCagA and GroEL IgG responses $(r=0.19, P<0.01$, data not shown).

\section{Correlation between $\mathrm{pCagA}$ and GroEL IgG responses with intensity of $O$. viverrini infection}

Among 437 O. viverrini negative and positive samples with EPG data, EPG ranged from 0 to 2,439 with a median of 10 eggs per gram. Most samples (97.2\%) showed light infections, with less than 500 EPG. In comparison with pCagA and GroEL IgG responses, no correlations were observed between EPG levels and pCagA IgG response $(P=0.49$, data not shown $)$ and between EPG levels and GroEL IgG response $(P=0.076$, data not shown).

\section{Association between seropositivity to $H$. pylori antigens and OV-associated HBA and CCA}

Multinomial logistic regression, adjusted for age and sex, showed that GroEL seropositivity was a risk factor for hepatobiliary abnormalities and CCA with adjusted odds ratios (95\% confidence intervals) of 2.11 (95\% CI $=1.20-3.71, P=0.008)$ and $2.13(95 \% \mathrm{CI}=1.21-3.75, P=0.009)$, respectively (Table 1). However, no such association was detected for pCagA.

\section{DISCUSSION}

The association between the 2 carcinogenic pathogens $O$. viverrini and $\mathrm{H}$. pylori is now established. The presence of $H$. pylori genes is associated with O. viverrini infection and its associated pathologies [7-9]. In this study, we further investigated the antibody response to the virulent factor CagA and immunogenic GroEL proteins of $H$. pylori by ELISA. These proteins were selected based on previous studies that shown CagA was a well-known virulent factor and immunogenic protein of $H$. pylori, and GroEL has been shown as a potential independent risk factor for gastritis, gastric cancer and other cancers [11, $12,14,15]$. Overall, the analysis showed that the IgG responses against CagA and GroEL were detected in all study groups. Besides, the response to these 2 antigens showed a weak positive yet significant correlation. These results indicated that the population was exposed to $H$. pylori infection.

Further analysis revealed that the level of antibodies to CagA protein and to GroEL showed no association to O. viverrini infection intensity. As more than $95 \%$ of the samples showed a 
Table 1. Association between lgG responses to Helicobacter pylori antigens and Opisthorchis viverrini-associated HBA and CCA

\begin{tabular}{lcccc}
\hline & $\begin{array}{c}\text { Control } \\
n=122\end{array}$ & $\begin{array}{c}\text { OV+HBA- } \\
n=140\end{array}$ & $\begin{array}{c}\text { OV+HBA+ } \\
n=144\end{array}$ & $\begin{array}{c}\text { CCA } \\
n=145\end{array}$ \\
\hline pCagA seropositive & $98(80 \%)$ & $112(80 \%)$ & $119(85 \%)$ & $117(80 \%)$ \\
OR & 1 & 1.03 & 1.22 & 1.07 \\
$95 \% \mathrm{Cl}$ & - & $0.56-1.88$ & $0.66-2.27$ & $0.58-1.96$ \\
$P$ & - & 0.92 & 0.51 & 0.8 \\
GroEL seropositive & $82(67 \%)$ & $98(70 \%)$ & $117(81 \%)$ & $118(81 \%)$ \\
OR & 1 & 1.13 & 2.11 & 2.13 \\
$95 \% \mathrm{Cl}$ & - & $0.67-1.92$ & $1.20-3.71$ & $1.21-3.75$ \\
$P$ & - & 0.62 & 0.009 & 0.008 \\
\hline
\end{tabular}

Odds ratios, 95\% confidence interval and $P$-value were calculated by multinomial logistic regression and significant $P$-values were noted in bold. ${ }^{a}$ Control=reference group; $\mathrm{OR}=$ odds ratio; $P=P$-value; $95 \% \mathrm{Cl}=95 \%$ confidence interval; $\mathrm{OV}=\mathrm{O}$. viverrini; $\mathrm{HBA}=$ hepatobiliary abnormalities; $\mathrm{CCA}=$ cholangiocarcinoma.

light infection, this is a limitation of this study. It could be interesting to test more samples from moderately and heavily OV infected patients.

Next, we investigated whether the antibody response to $H$. pylori is associated with the pathological consequences of $O$. viverrini. In contrast to $O$. viverrini infection intensity, $\mathrm{HBA}$ and CCA were associated with antibody to GroEL; however, no such association was detected for CagA protein. GroEL is one of the most abundant proteins and expressed in most strains of $H$. pylori [23]. It was detected on the outside surface of the bacterium where it is likely exposed to the immune response [24]. Indeed, H. pylori GroEL was shown to activate NF- $\mathrm{kB}$ and; thus, induce IL-8 production through TLR-2 and -4 associated pathways in gastric epithelial cells [25]. Pathogenesis of O. viverrini-associated hepatobiliary diseases are proposed as an inflammation induced process, and co-infection with $H$. pylori enhances the severity of $O$. viverrini infection [10]. Taken together, the immunogenic abundant GroEL might further enhance the inflammation and tissue damages, which in turn accelerate the carcinogenesis of O. viverrini-associated CCA.

Another interesting point of this study was that there was no association between CagA seropositivity and HBA. This finding contradicts results from a previous study showing that cagA gene is associated with HBA [8]. This discrepancy might be explained by gene expression variation of cagA gene due to the genetic heterogeneity of promoter region [26], gene motifs [27], and copy number [28]. These genetic variations might cause the differential gene expression of $c a g A$, which in turn affects the immune response. However, this hypothesis warrants further investigation.

The limitation of this study was that we looked at the response to only 2 proteins of $H$. pylori. Several other proteins have been involved in pathogenesis and are also biomarkers of $H$. pylori and its associated diseases [11]. We believed that employing a multiplex ELISA in a population-based study with a higher number of samples may provide a better understanding of the complex interaction between these 2 carcinogenic pathogens. Another issue that needs to be considered is that this study is not a longitudinal study. Therefore, it cannot answer whether $\mathrm{H}$. pylori is the cause or the effect of HBA and CCA. Further studies are certainly needed to clarify this statement.

In conclusion, the results showed that GroEL seropositivity was a risk factor for both HBA and CCA. Further investigations regarding the potential use of GroEL as a biomarker for O. viverrini-associated diseases and the role of this protein in pathogenesis is needed, as the incidence of CCA in the northeast region of Thailand is still the highest worldwide.

\section{ACKNOWLEDGMENTS}

This work was supported by the Thailand Research Fund Senior Scholar (RTA 5680006). BS is a TRF Senior Research Scholar. IJ received a scholarship from the Faculty of Medicine, Khon Kaen University. We thank Professor Yukifuma Nawa for numerous discussions and for his assistance in obtaining the commercial antibody determination kit E-Plate Eiken $H$. pylori antibody (Eiken Chemical Co., Ltd., Tokyo, Japan). We acknowledge the advice of Professors Ratha-Korn Vilaichone (Thammasat University) and Veerasak Punyapornwithaya (Chiang Mai University). We also thank Manop Sripa (Khon Kaen University) for his technical assistance. 


\section{CONFLICT OF INTEREST}

The authors declare no potential conflicts of interest.

\section{REFERENCES}

1. Elkins DB, Haswell-Elkins MR, Mairiang E, Mairiang P, Sithithaworn P, Kaewkes S, Bhudhisawasdi V, Uttaravichien T. A high frequency of hepatobiliary disease and suspected cholangiocarcinoma associated with heavy Opisthorchis viverrini infection in a small community in north-east Thailand. Trans R Soc Trop Med Hyg 1990; 84: 715-719. https://doi.org/10.1016/0035-9203(90)90159-c

2. Sripa B, Kaewkes S, Sithithaworn P, Mairiang E, Laha T, Smout M, Pairojkul C, Bhudhisawasdi V, Tesana S, Thinkamrop B, Bethony JM, Loukas A, Brindley PJ. Liver fluke induces cholangiocarcinoma. PLoS Med 2007; 4: e201. https://doi.org/10.1371/journal. pmed.0040201

3. Banales JM, Cardinale V, Carpino G, Marzioni M, Andersen JB, Invernizzi P, Lind GE, Folseraas T, Forbes SJ, Fouassier L, Geier A, Calvisi DF, Mertens JC, Trauner M, Benedetti A, Maroni L, Vaquero J, Macias RI, Raggi C, Perugorria MJ, Gaudio E, Boberg KM, Marin JJ, Alvaro D. Cholangiocarcinoma: current knowledge and future perspectives consensus statement from the European Network for the Study of Cholangiocarcinoma (ENSCCA). Nat Rev Gastroenterol Hepatol 2016; 13: 261-280. https:// doi.org/10.1038/nrgastro.2016.51

4. Kamsa-Ard S, Luvira V, Suwanrungruang K, Kamsa-Ard S, Luvira V, Santong C, Srisuk T, Pugkhem A, Bhudhisawasdi V, Pairojkul C. Cholangiocarcinoma trends, incidence, and relative survival in Khon Kaen, Thailand from 1989 through 2013: a populationbased cancer registry study. J Epidemiol 2018; 29: 197-204. https://doi.org/10.2188/jea.je20180007

5. Rizvi S, Khan SA, Hallemeier CL, Kelley RK, Gores GJ. Cholangiocarcinoma - evolving concepts and therapeutic strategies. Nat Rev Clin Oncol 2018; 15: 95-111. https://doi.org/10.1038/nrclinonc. 2017.157

6. Nilsson HO, Mulchandani R, Tranberg KG, Stenram U, Wadström T. Helicobacter species identified in liver from patients with cholangiocarcinoma and hepatocellular carcinoma. Gastroenterology 2001; 120: 323-324. https://doi.org/10.1053/gast. 2001.21382

7. Deenonpoe R, Chomvarin C, Pairojkul C, Chamgramol Y, Loukas A, Brindley PJ, Sripa B. The carcinogenic liver fluke Opisthorchis viverrini is a reservoir for species of Helicobacter. Asian Pac J Cancer Prev 2015; 16: 1751-1758. https://doi.org/10.7314/apjcp. 2015.16.5.1751

8. Deenonpoe R, Mairiang E, Mairiang P, Pairojkul C, Chamgramol Y, Rinaldi G, Loukas A, Brindley PJ, Sripa B. Elevated prevalence of Helicobacter species and virulence factors in opisthorchiasis and associated hepatobiliary disease. Sci Rep 2017; 7: 42744. https://doi.org/10.1038/srep42744

9. Dangtakot R, Pinlaor S, Itthitaetrakool U, Chaidee A, Chomva- rin C, Sangka A, Wilailuckana C, Pinlaor P. Co-infection of Helicobacter pylori and Opisthorchis viverrini enhances the severity of hepatobiliary abnormalities in hamsters. Infect Immun 2017; 85: e00009-17. https://doi.org/10.1128/iai.00009-17

10. Sripa B, Tangkawattana S, Brindley PJ. Update on pathogenesis of opisthorchiasis and cholangiocarcinoma. Adv Parasitol 2018; 102: 97-113. https://doi.org/10.1016/bs.apar.2018.10.001

11. Gao L, Michel A, Weck MN, Arndt V, Pawlita M, Brenner H. Helicobacter pylori infection and gastric cancer risk: evaluation of 15 H. pylori proteins determined by novel multiplex serology. Cancer Res 2009; 69: 6164-6170. https://doi.org/10.1158/00085472.can-09-0596

12. Song H, Michel A, Nyrén O, Ekström AM, Pawlita M, Ye W. A CagA-independent cluster of antigens related to the risk of noncardia gastric cancer: Associations between Helicobacter pylori antibodies and gastric adenocarcinoma explored by multiplex serology. Int J Cancer 2014; 134: 2942-2950. https://doi.org/ 10.1002/ijc.28621

13. Cai H, Ye F, Michel A, Murphy G, Sasazuki S, Taylor PR, Qiao YL, Park SK, Yoo KY, Jee SH, Cho ER, Kim J, Chen SC, Abnet CC, Tsugane S, Cai Q, Shu XO, Zheng W, Pawlita M, Epplein M. Helicobacter pylori blood biomarker for gastric cancer risk in East Asia. Int J Epidemiol 2016; 45: 774-781. https://doi.org/10. 1093/ije/dyw078

14. Blase JL, Campbell PT, Gapstur SM, Pawlita M, Michel A, Waterboer T, Teras LR. Prediagnostic Helicobacter pylori antibodies and colorectal cancer risk in an elderly, caucasian population. Helicobacter 2016; 21: 488-492. https://doi.org/10.1111/hel.12305

15. Fernández de Larrea-Baz N, Michel A, Romero B, Pérez-Gómez B, Moreno V, Martín V, Dierssen-Sotos T, Jiménez-Moleón JJ, Castilla J, Tardón A, Ruiz I, Peiró R, Tejada A, Chirlaque MD, Butt JA, Olmedo-Requena R, Gómez-Acebo I, Linares P, Boldo E, Castells A, Pawlita M, Castaño-Vinyals G, Kogevinas M, de Sanjosé S, Pollán M, Del Campo R, Waterboer T, Aragonés N. Helicobacter pylori antibody reactivities and colorectal cancer risk in a case-control study in Spain. Front Microbiol 2017; 8: 888. https://doi.org/10.3389/ fmicb.2017.00888

16. Murphy G, Michel A, Taylor PR, Albanes D, Weinstein SJ, Virtamo J, Parisi D, Snyder K, Butt J, McGlynn KA, Koshiol J, Pawlita M, Lai GY, Abnet CC, Dawsey SM, Freedman ND. Association of seropositivity to Helicobacter species and biliary tract cancer in the ATBC study. Hepatology 2014; 60: 1963-1971. https://doi. org/10.1002/hep.27193

17. Sripa B, Mairiang E, Thinkhamrop B, Laha T, Kaewkes S, Sithithaworn P, Tessana S, Loukas A, Brindley PJ, Bethony JM. Advanced periductal fibrosis from infection with the carcinogenic human liver fluke Opisthorchis viverrini correlates with elevated levels of interleukin-6. Hepatology 2009; 50: 1273-1281. https:// doi.org/10.1002/hep.23134

18. RStudio Team. RStudio: Integrated Development for R. [Internet]; [cited 2018 Sept 17]. Available from: https://www.rstudio. $\mathrm{com} /$

19. The R Core Team. Package "stats." R: A language and environ- 
ment for statistical computing R Foundation for Statistical Computing [Internet]; 2001 [cited 2018 Aug 10]; Available from: https://www.rdocumentation.org/packages/stats/versions/3.5.1

20. Hijmans RJ, van Etter J, Sumner M, Cheng J, Baston D, Bevan A, Bivand R, Busetto L, Canty M, Fasoli B, Forrest D, Ghosh A, Golicher D, Gray J, Greenberg JA, Hiemstra P, Hingee K, Institute for Mathematics Applied Geosciences, Karney C, Mattiuzzi M, Mosher S, Naimi B, Nowosad J, Pebesma E, Lamigueiro OP, Racine EB, Rowlingson B, Shortridge A, Venables B, Wueest R. Package "raster." [Internet]; Available from: https://cran.r-project. org/web/packages/raster/raster.pdf

21. Wickham H, Chang W, Henry L. Package 'ggplot2.' R: A language and environment for statistical computing R Foundation for Statistical Computing [Internet]; Available from: https://cran. r-project.org/web/packages/ggplot2/index.html

22. Ripley B, Venables W. Package "nnet." R: A language and environment for statistical computing R Foundation for Statistical Computing [Internet]; Available from: http://www.stats.ox. ac.uk/pub/MASS4/

23. Jungblut PR, Bumann D, Haas G, Zimny-Arndt U, Holland P, Lamer S, Siejak F, Aebischer A, Meyer TF. Comparative proteome analysis of Helicobacter pylori. Mol Microbiol 2000; 36: 710-725. https://doi.org/10.1046/j.1365-2958.2000.01896.x

24. Kamiya S, Yamaguchi H, Osaki T, Taguchi H. A virulence factor of
Helicobacter pylori: role of heat shock protein in mucosal inflammation after H. pylori infection. J Clin Gastroenterol 1998; 27 (suppl): 35-39. https://doi.org/10.1097/00004836-19980000100007

25. Takenaka R, Yokota K, Ayada K, Mizuno M, Zhao Y, Fujinami Y, Lin SN, Toyokawa T, Okada H, Shiratori Y, Oguma K. Helicobacter pylori heat-shock protein 60 induces inflammatory responses through the Toll-like receptor-triggered pathway in cultured human gastric epithelial cells. Microbiology 2004; 150: 3913-3922. https://doi.org/10.1099/mic.0.27527-0

26. Ferreira RM, Pinto-Ribeiro I, Wen X, Marcos-Pinto R, Dinis-Ribeiro M, Carneiro F, Figueiredo C. Helicobacter pylori cagA promoter region sequences influence CagA expression and interleukin 8 secretion. J Infect Dis 2016; 213: 669-673. https://doi.org/ 10.1093/infdis/jiv467

27. Ahire D, Alston T, Raffaniello R. Variations in the multimerization region of the Helicobacter pylori cytotoxin CagA affect virulence. Oncol Lett 2017; 13: 1444-1450. https://doi.org/10.3892/ ol.2017.5562

28. Jang S, Su H, Blum FC, Bae S, Choi YH, Kim A, Hong YA, Kim J, Kim JH, Gunawardhana N, Jeon YE, Yoo YJ, Merrell DS, Ge L, Cha JH. Dynamic expansion and contraction of cagA copy number in Helicobacter pylori impact development of gastric disease. mBio 2017; 8: e01779-16. https://doi.org/10.1128/mbio.01779-16 